\title{
ON A LINEAR AGE-DEPENDENT POPULATION DIFFUSION MODEL*
}

\author{
BY \\ MICHEL R. LANGLAIS \\ Purdue University
}

1. Introduction. We discuss here a model described by Gurtin [3] for age-dependent populations with diffusion in a bounded set $\Omega$ of $\mathbb{R}^{\mathbf{N}}$.

In this linear theory the age-space structure is studied through the population distribution $u(t, a, x)$ where $t$ is a time, $a$ age $(0<a<A)$ and $x$ spatial position. The evolution of $u$ is governed by the equation (balance law):

$$
\frac{\partial u}{\partial t}+\frac{\partial u}{\partial a}+\mu u-\Delta \int_{0}^{A} k(a, \alpha) u(t, \alpha, x) d \alpha=0
$$

where $\mu=\mu(t, a, x)$ is the death-modulus and $\nabla \int_{0}^{A} k(a, \alpha) u(t, \alpha, x) d \alpha$ is the flux of population by spatial diffusion. Here $\Delta$ is the Laplacian and $\nabla$ is the gradient in $\mathbb{R}^{\mathrm{N}}$. The reader is referred to Hoppensteadt [6] for equations of this form, but without diffusion.

We assume that the birth process is given by the birth law:

$$
u(t, 0, x)=\int_{0}^{A} \beta(t, a, x) u(t, a, x) d a
$$

( $\beta$ is the birth modulus), that there is no diffusion through the boundary $\partial \Omega$ of $\Omega$, that is:

$$
\frac{\partial}{\partial \eta} \int_{0}^{A} k(a, \alpha) u(t, \alpha, x) d \alpha=0
$$

where $\partial / \partial \eta$ is the normal derivative, and that the initial population is known:

$$
u(0, a, x)=u_{0}(a, x) \text {. }
$$

$A$ is the maximum life expectancy of the species.

The initial boundary value problem (1)-(4) will be referred to as problem (I), namely (subscripts indicate partial differentiation):

$$
\begin{array}{ll}
u_{t}+u_{a}+\mu u-\Delta \int_{0}^{A} k(a, \alpha) u(t, \alpha, x) d \alpha=0, & t>0,0<a<A, x \in \Omega, \\
u(0, a, x)=u_{0}(a, x), & 0<a<A, x \in \Omega, \\
u(t, 0, x)=\int_{0}^{A} \beta(t, a, x) u(t, a, x) d a, & t>0, x \in \Omega, \\
\frac{\partial}{\partial \eta} \int_{0}^{A} k(a, \alpha) u(t, \alpha, x) d \alpha=0, & t>0,0<a<A, x \in \Omega .
\end{array}
$$

* Received November 23, 1981; revised version received January 19, 1982. Author's permanent address: U. E. R. de Mathematiques et Informatique, Universite de Bordeaux 1, 33405 Talence Cedex France. 
In the particular case when the kernel $k$ is independent of the variable $\alpha$, the equation (1) and the boundary condition (3) can be expressed in a simpler form and problem (I) becomes problem (II), that is:

$$
\begin{array}{cc}
u_{t}+u_{a}+\mu u-k(a) \Delta \int_{0}^{A} u(t, \alpha, x) d \alpha=0, t>0, & 0<a<A, x \in \Omega, \\
u(0, a, x)=u_{0}(a, x), & 0<a<A, x \in \Omega, \\
u(t, 0, x)=\int_{0}^{A} \beta(t, a, x) u(t, a, x) d a, & t>0, x \in \Omega, \\
\frac{\partial}{\partial \eta} \int_{0}^{A} u(t, a, x) d a=0, & t>0, x \in \partial \Omega .
\end{array}
$$

Some results concerning problem (I) are given in di Blasio and Lamberti [1]. The method emphasized here (initiated in Langlais [8]) is quite different. Under suitable assumptions this method provides existence and uniqueness in problem (II) (which is the first model derived in [3]), and is helpful for the nonlinear model investigated in Garroni and Langlais [2]. On the other hand, the hypotheses needed to solve problem (II) when $\mu$ is not bounded appear again in the model with nonlinear diffusion studied by Langlais [9], and we expect them to be useful in the nonlinear diffusion model described in Gurtin and MacCamy [4, 5] (investigated for constant $\mu$ by MacCamy [11]). In these two linear models the solution can become negative in a finite time (see $[4,9]$ ). This paper is a first step towards nonlinear models.

2. Notation and basic assumptions. $T$ and $A$ are positive and finite real numbers; $\Omega$ is a bounded open set of $\mathbb{R}^{\mathrm{N}}$ with smooth boundary $\partial \Omega$. The variables $t, a$ and $x$ lie respectively in $(0, T),(0, A)$ and $\Omega$. The set $(0, T) \times(0, A)$ is denoted $\mathcal{O}$ and $Q$ is the product $\mathcal{O} \times \Omega$.

Let $\mu$ be a real-valued function on $Q$ satisfying:

$$
\begin{aligned}
& \mu \text { is continuous on }[0, T] \times[0, A) \times \bar{\Omega} ; \\
& \mu(t, a, x) \geq 0 \text { on } Q ; \\
& \nabla \mu \text { is bounded on } Q .
\end{aligned}
$$

$\mu$ is not and will not be assumed to be bounded near $a=A$ (see further remarks).

We suppose that $\beta$ is a real-valued function on $Q$ such that:

$\beta$ is bounded on $Q$;

there exists a contant $C_{1}$ such that

$$
\int_{0}^{A}\left[\beta^{2}+|\nabla \beta|^{2}\right](t, a, x) d a \leq C_{1} \text { in }(0, T) \times \Omega .
$$

The kernel $k$ is a real measurable function defined in $(0, A) \times(0, A)$; in problem (I) we shall suppose:

there exists a constant $C_{2}$ such that for each $\varphi \in L^{2}(0, A)$ :

$$
\int_{0}^{1}\left[\int_{0}^{A} k(a, \alpha) \varphi(\alpha) d \alpha\right]^{2} d \alpha \leq C_{2}^{2} \int_{0}^{1} \varphi^{2}(a) d a:
$$


for each $\varphi \in L^{2}(0, A)$ we have:

$$
\int_{0}^{A} \int_{0}^{A} k(a, \alpha) \varphi(\alpha) \varphi(a) d a d \alpha \geq 0 .
$$

The first part of $(k)^{1}$ means that the map:

$$
\varphi \in L^{2}(0, A) \rightarrow \int_{0}^{A} k(a, \alpha) \varphi(\alpha) d \alpha \in L^{2}(0, A)
$$

is continuous. The last part of $(\mathbf{k})^{1}$ is a monotonicity-type condition used in [1].

In the particular case where the kernel $k$ does not depend on $\alpha$ we shall simply need:

$$
k \in L^{2}(0, A) ; \quad k(a) \geq 0 \quad \text { in }(0, A) .
$$

Now if $U$ is any open set of either $\mathbb{R}$ or $\mathbb{R}^{2}$ and if $H$ is any of the Sobolev spaces of order 1 or $2 H^{1}(\Omega)$ and $H^{2}(\Omega)$, then $L^{2}(U, H)$ is the Hilbert space of measurable and square integral function $v: U \rightarrow H$ (see Lions and Magenes [10]).

3. Statement of results. The first result concerns problem (I), that is the initial boundary value problem (1)-(4).

Theorem 1. Let $(\mu, \beta, k)$ satisfy $(\mu)^{1},(\beta)^{1},(\mathbf{k})^{1}$ and

$$
\begin{aligned}
& \Delta \mu \in L^{\infty}(Q), \quad \partial \mu / \partial \eta=0 \text { in } \mathcal{O} \times \partial \Omega ; \\
& \int_{0}^{A}|\Delta \beta|^{2}(t, a, x) d a \leq C_{1} \text { in }(0, T) \times \Omega, \quad \frac{\partial \beta}{\partial \eta}=0 \text { in } \mathcal{O} \times \partial \Omega .
\end{aligned}
$$

Then given any $u_{0}$ in $L^{2}\left(0, A ; H^{2}(\Omega)\right)$ such that $\partial u_{0} / \partial \eta=0$ in $(0, A) \times \partial \Omega$ there exists a unique $u$ belonging to $L^{2}\left(\mathcal{O} ; H^{2}(\Omega)\right.$, a solution to problem (I) and verifying $\partial u / \partial \eta=0$ in $\mathcal{O} \times \partial \Omega$.

It is worth while to notice that the initial boundary conditions (2-4) make sense provided the solution belongs to $L^{2}\left(\mathcal{O} ; H^{2}(\Omega)\right)$.

As the solution $u$ lies in $L^{2}\left(\mathcal{O} ; H^{2}(\Omega)\right)$, the properties of $k$ ensure that $\int_{0}^{A} k(a, \alpha) u(t, \alpha$, $x) d \alpha$ lies in $L^{2}\left(\mathcal{O} ; H^{2}(\Omega)\right)$; hence the Neumann boundary condition (3) makes sense and is fulfilled because $u$ satisfies the homogeneous boundary Neumann condition. Moreover, from Eq. (1) we deduce that $u_{t}+u_{a}+\mu \cdot u$ belongs to $L^{2}(Q)$. Now take any $A_{0}, 0<A_{0}<$ $A$, and set $\mathcal{O}_{0}=(0, T) \times\left(0, A_{0}\right), Q_{0}=\mathcal{O}_{0} \times \Omega$; then $\mu$ is bounded on $Q_{0}$ and $u_{t}+u_{a}$ lies in $L^{2}\left(Q_{0}\right)$. Thus bearing in mind that $u$ is in $L^{2}\left(Q_{0}\right)$, initial conditions (2) and (4) make sense.

When the data $u_{0}$ is not smooth enough or when the conditions $(\mu)^{2}$ and $(\beta)^{2}$ are not fulfilled we can prove the existence and uniqueness of a suitable weak solution. Hence Theorem 1 can be considered as a regularity theorem. But it is more interesting to view it as a basic result from which we can derive particular cases.

Let us assume first that:

$$
k(a, \alpha)=h(a) h(\alpha), \quad h \in L^{2}(0, A) .
$$

This assumption is stronger that $(\mathrm{k})^{1}$. The Newmann boundary condition (3) becomes:

$$
\frac{\partial}{\partial \eta} \int_{0}^{A} h(\alpha) u(t, \alpha, x) d \alpha=0 \text { in }(0, T) \times \partial \Omega .
$$

Condition $(\mathbf{k})^{3}$ allows us to remove $(\mu)^{2}$ and $(\beta)^{2}$ in Theorem 1. 
Theorem 2. Suppose that $(\mu)^{1},(\beta)^{1},(\mathrm{k})^{3}$ are satisfied; then given any $u_{0}$ in $L^{2}\left(0, A ; H^{1}(\Omega)\right)$ there exists a unique $u$ in $L^{2}\left(\mathcal{O} ; H^{1}(\Omega)\right)$, a solution to problem (I) and such that:

$$
\int_{0}^{A} h(\alpha) u(t, \alpha, x) d \alpha \in L^{2}\left(0 . T ; H^{2}(\Omega)\right) .
$$

We investigate now the particular case when the kernel $k$ is independent of $\alpha$, that is, problem (II). Assume first that $k$ lies in $C^{1}([0, A])$ and that:

there exists a real constant $k_{0}$ such that $k(a) \geq k_{0}>0$ in $(0, A)$

If we let $u(t, a, x)=k(a) v(t, a, x)$ in $Q$, then, at least formally, $v$ is a solution of the equations:

$$
\begin{aligned}
& k\left(v_{t}+v_{a}\right)+\left(\mu k+k_{a}\right) v-k \Delta \int_{0}^{A} k(\alpha) v(t, \alpha, x) d \alpha=0 \text { in } Q, \\
& v(0, a, x)=[k(a)]^{-1} u_{0}(a, x) \text { in }(0, A) \times \Omega \\
& v(t, 0, x)=[k(0)]^{-1} \int_{0}^{A} \beta(t, a, x) k(a) v(t, a, x) d a \text { on }(0, T) \times \Omega, \\
& \frac{\partial}{\partial \eta} \int_{0}^{A} k(a) v(t, a, x) d a=0 \text { on } \mathcal{O} \times \partial \Omega .
\end{aligned}
$$

This boundary-value problem with $(k)^{4}$ and the problem (I) with $(k)^{3}$ have the same qualitatives properties. We can prove that when $(\mu)^{1},(\beta)^{1},(\mathrm{k})^{2}$ and $(\mathrm{k})^{4}$ are satisfied for any $u_{0}$ given in $L^{2}\left(0, A ; H^{1}(\Omega)\right)$ there exists a unique $v$ verifying (6)- see Theorem 2-a solution to problem (III). From $v$ we get $u$, a solution to problem (II).

Remark 1. Up to now we did not suppose $\mu$ to be bounded at $a=A\left(\operatorname{see}(\mu)^{1}\right)$. Actually if $\mu$ is rapidly increasing at $a=A$ then any of the solutions whose existence has been previously established vanishes at $a=A$. More precisely, the conditions $u(t, A$, $x)=0$ in $(0, T) \times \Omega$ and the two conditions:

$$
\begin{gathered}
0<t<A, \quad x \in \Omega \lim _{a \rightarrow A} \int_{0}^{t} \mu(\tau, a-t+\tau, x) d t=+\infty \\
A<t<T, \quad x \in \Omega \lim _{a \rightarrow A} \int \mu(t-a+\alpha, \alpha, x) d \alpha=+\infty
\end{gathered}
$$

are equivalent (see Langhaar [7], [2] and [8]: this property is independent of the diffusion term in (1)).

Unfortunately, we have not been able to solve Problem (II) when $(\mu)^{3}$ is fulfilled (except when $(\mathbf{k})^{4}$ is true) without additional hypotheses on $u, k$ and $u_{0}$.

THEOREM 3. Let $(\mu)^{1},(\beta)^{1},(\mathrm{k})^{2}$ be satisfied. Let $u_{0}$ be in $L^{2}((0, A) \times \Omega)$ and verify $\int_{0}^{A} u_{0}(a$, x) $d a \in H^{1}(\Omega)$. Assume either:

there exist two constants $\lambda_{1}, \lambda_{2}$ such that

$$
\begin{aligned}
& \mu^{2}-\mu_{t}-\mu_{a} \geq \lambda_{1} \mu+\lambda_{2} \text { on } Q ; \\
& \mu(0, a, x) u_{0} \in L^{2}((0, A) \times \Omega) ;
\end{aligned}
$$


there exists a constant $M$ such that

$$
\int_{0}^{A} \mu^{2}(t, a, x) k^{2}(a) d a \leq M \text { on }(0, T) \times \Omega ;
$$

or:

$$
\text { there exists a constant } m \text { such that } \int_{0}^{A} \mu(t, a, x) d a \leq m \text { in }(0, T) \times \Omega . \quad(\mu)^{5}
$$

Then there exists a unique $u$ in $L^{2}(Q)$ satisfying $(6)-\mu u \in L^{2}(Q)$ when $(\mu)^{4}$ is true-a solution to problem (II).

Remark 2. When $(\mu)^{3}$ is satisfied and when $k$ is a constant then $(\mu)^{4}($ iii) is not fulfilled; nevertheless, to conclude we merely apply Theorem 2 . More generally, $(k)^{4},(\mu)^{3}$, and $(\mu)^{4}$ (iii), are not consistent, but we have previously dealt with $(\mathrm{k})^{4}$.

The conditions $(\mu)^{3}$ and $(\mu)^{4}(\mathrm{i})$ are realized when:

$$
\begin{gathered}
\mu(a)=q(A-a)^{-p}, \quad q>0 \text { and } p>1 \text { or } q \geq 1 \text { and } p=1, \\
\mu(a)=q \exp +1 / A-a, \quad q>0 .
\end{gathered}
$$

4. Proofs. We first discuss a preliminary result from which Theorem 1 is proved, for bounded $\mu$, using a fixed-point method. Then we turn to the general case. Theorem 3 is proved along the same lines, but we shall point out the differences in the first and last steps. The proof of Theorem 2 is omitted.

Now let $u$ be a solution to problem (I) or problem (II) and set:

$$
u(t, a, x)=e^{\lambda t} v(t, a, x) \text { in } Q, \lambda \text { constant; }
$$

then $v$ is the solution to problem (I) or problem (II) with $\mu$ replaced by $\mu+\lambda$. This change of unknown function will be done throughout this section, for suitable positive values of $\lambda$, and $v$ will be simply denoted $u$.

4-1. Preliminary results. We investigate the folowing initial-boundary value problem:

$$
\begin{aligned}
& u_{t}+u_{a}+\lambda u-\Delta \int_{0}^{A} k(\alpha, a) u(t, \alpha, x) d \alpha=f \text { in } Q, \\
& u(0, a, x)=u_{0}(a, x) \text { in }(0, A) \times \Omega, \\
& u(t, 0, x)=b(t, x) \text { in }(0, T) \times \Omega, \\
& \frac{\partial}{\partial \eta} \int_{0}^{A} k(a, \alpha) u(t, \alpha, x) d a=0 \text { in } \mathcal{O} \times \partial \Omega .
\end{aligned}
$$

Here $\lambda$ is a positive constant. In order to get the existence of a solution we introduce the eigenfunctions of the Neumann problem in $\Omega$ :

$$
\begin{gathered}
-\Delta w_{j}=v_{j} w_{j} \text { in } \Omega, \quad \partial w_{j} / \partial \eta=0 \text { in } \partial \Omega, \\
\int_{\Omega} w_{j}^{2}=1, \quad \int_{\Omega} w_{j} \cdot w_{i} d x=0 \quad j \neq i .
\end{gathered}
$$


Expressing the data on the form:

$$
\begin{gathered}
f(t, a, x)=\sum_{j} f_{j}(t, a) w_{j}(x), \\
b(t, x)=\sum_{j} b_{j}(t) w_{j}(x), \quad u_{0}(a, x)=\sum_{j} u_{0, j}(a) w_{j}(x),
\end{gathered}
$$

it is appealing to seek as a formal solution to problem (III) the series

$$
u(t, a, x)=\sum_{j} u_{j}(t, a) w_{j}(x) .
$$

It turns out that for suitable data $\left(f, b, u_{0}\right)$ this gives a solution.

TheOREM 4. Let $k$ satisfy $(\mathbf{k})^{1}$ and let $\left(f, b, u_{0}\right)$ be such that:

$$
\begin{aligned}
f \in L^{2}\left(\mathcal{O} ; H^{2}(\Omega)\right), & & \partial f / \partial \eta=0 \text { in } \mathcal{O} \times \partial \Omega, \\
b \in L^{2}\left(0, T ; H^{2}(\Omega)\right), & & \partial b / \partial \eta=0 \text { in }(0, T) \times \partial \Omega, \\
u_{0} \in L^{2}\left(0, T ; H^{2}(\Omega)\right), & & \partial u_{0} / \partial \eta=0 \text { in }(0, \mathrm{~A}) \times \partial \Omega .
\end{aligned}
$$

Then there exists a unique solution $u$ in $L^{2}\left(\mathcal{O} ; H^{2}(\Omega)\right)$ to problem (IV). Moreover, this solution satisfies the boundary condition:

$$
\partial u / \partial \eta=0 \text { on } \mathcal{O} \times \partial \Omega
$$

and the a priori estimates:

$\lambda \int_{Q}\left[u^{2}+|\nabla u|^{2}\right] d t d a d x \leq \frac{1}{2} \int_{(0, A) \times \Omega}\left[u_{0}^{2}+\left|\nabla u_{0}\right|^{2}\right] d a d x$

$$
+\frac{1}{2} \int_{(0, T) \times \Omega}\left[b^{2}+|\nabla b|^{2} d t d x+\int_{Q}[f \cdot u+\nabla f \cdot \nabla u] d t d a d x,\right.
$$

$\lambda \int_{Q}|\Delta u|^{2} d t d a d x \leq \frac{1}{2} \int_{(0, A) \times \Omega}\left|\Delta u_{0}\right|^{2} d a d x$

$$
+\frac{1}{2} \int_{(0, T) \times \Omega}|\Delta b|^{2} d a d x+\int_{Q} \Delta f \cdot \Delta u d t d a d x
$$

Proof. The existence of a solution is obtained by the method of separation of variables outlined above. The solution satisfies the estimates (9) and (10).

Now let $u^{1}$ and $u^{2}$ be solutions of (IV). The difference $u=u^{1}-u^{2}$ is solution of the same problem (IV) with $f=0, b=0$ and $u_{0}=0$. Multiplying equation (IV) ${ }^{1}$ by $u$ and integrating over $Q$ yields:

$\int_{o}\left(u_{t}+u_{a}\right) u d t d a d x+\lambda \int_{Q} u^{2} d t d a d x-\int_{Q} \Delta \int_{0}^{A} k(a, \alpha) u(t, a, x) d \alpha \cdot u d t d a d x=0$

Integrating by parts the last term and using $(\mathrm{k})^{1}$ we have:

$$
\begin{aligned}
\int_{Q} \nabla \int_{V}^{A} k(a, \alpha) u(t, \alpha, x) d \alpha & \cdot \nabla u(t, a, x) d t d a d x \\
= & \int_{(0, T) \times \Omega}\left[\int_{0}^{A} \int_{0}^{A} k(a, \alpha) \nabla u(t, \alpha, x) \cdot \nabla u(t, a, x) d \alpha d a\right] d t d x \geq 0
\end{aligned}
$$


$u$ belonging to $L^{2}\left(\mathcal{O} ; H^{2}(\Omega)\right)$. From equation (IV) ${ }^{1}$ we get $u_{t}+u_{a} \in L^{2}(Q)$. We can integrate by parts the first term in (11); this gives:

$$
\int_{Q}\left(u_{t}+u_{a}\right) u d t d a d x=\frac{1}{2}\left[\int_{(0, A) \times \Omega} u^{2}(T, a, x) d a d x+\int_{(0, T) \times \Omega} u^{2}(t, A, x) d t d x\right] \geq 0,
$$

because $u$ vanishes on $t=0$ and $a=0$. It is easy now to prove that $u=0$; from (11) we derive $\int_{Q} u^{2}(t, a, x) d t d a d x \leq 0$.

4.2 Proof of Theorem 1 for bounded $\mu$. We assume that $\mu$ is bounded. Let $\lambda$ be a positive constant large enough compared with $C_{i}$ (see the hypotheses on $\beta$ ), with the norms of $\mu$ and $\Delta \mu$ in $L^{\infty}(Q)$ and with the norms of $\nabla \mu$ in $\left[L^{\infty}(Q)\right]^{N}$. We deal with $v=e^{-\lambda t} u$ still denoted $u$.

We want to prove Theorem 1 using a fixed-point method and (IV), (9), (10). Let $E$ be the Hilbert space:

$$
F=\left\{u \in L^{2}\left(\mathcal{O} ; H^{2}(\Omega)\right), \partial u / \partial \eta=0 \text { in } \mathcal{O} \times \partial \Omega\right\} .
$$

Assuming that the hypotheses of theorem 1 ( $\mu$ bounded) are satisfied, for any $w$ in $E$ there exists a unique $u=S w$ in $E$, a solution to

$$
\begin{aligned}
& u_{t}+u_{a}+\lambda u-\Delta \int_{0}^{A} k(a, \alpha) u(t, \alpha, x) d \alpha=-\mu w \text { in } Q, \\
& u(0, a, x)=u_{0}(a, x) \text { in }(0, A) \times \Omega, \\
& u(t, 0, x)=\int_{0}^{A} \beta(t, a, x) w(t, a, x) d a \text { in }(0, T) \times \Omega, \\
& \frac{\partial}{\partial \eta} \int_{0}^{A} k(a, \alpha) u(t, \alpha, x) d \alpha=0 \text { in } \mathcal{O} \times \partial \Omega .
\end{aligned}
$$

Thus we define a map $S: E \rightarrow E$. Its fixed points are the solutions to problem (I) in $E$. From (9) and (10) we deduce that $S$ is continuous.

Let $w^{1}\left(\right.$ resp. $\left.w^{2}\right)$ be in $E$ and set $u^{1}=S w^{1}\left(\right.$ resp. $\left.u^{2}=S w^{2}\right)$. The function $u=u^{1}-u^{2}$ is a solution to problem (IV) with:

$$
\begin{aligned}
f & =-\mu w \text { in } Q, \quad w=w^{1}-w^{2} \text { in } Q . \\
b(t, x) & =\int_{0}^{A} \beta(t, a, x) w(t, a, x) d a \text { in }(0, T) \times \Omega, \\
u_{0}(a, x) & =0 \text { in }(0, A) \times \Omega .
\end{aligned}
$$

Before employing the estimates (9) (10), we need to bound the right-hand side of (9), (10) when $f, b$ and $u_{0}$ are given by (12).

First, the properties of $\beta$-that is, $(\beta)^{1},(\beta)^{2}$-give:

$$
\begin{aligned}
\int_{(0, T) \times \Omega}\left[b^{2}+|\nabla b|^{2}\right] d t d x & \leq 3 C_{1} \int_{Q}\left[w^{2}+|\nabla w|^{2}\right] d t d a d x, \\
\int_{(0, T) \times \Omega}|\Delta b|^{2} d t d x & \leq 4 C_{1} \int_{Q}\left[w^{2}+|\nabla w|^{2}+|\Delta w|^{2}\right] d t d a d x .
\end{aligned}
$$


Here we used the Holder inequality. The same calculation for $\mu$ leads to:

$$
\begin{gathered}
\int_{Q}[f \cdot u+\nabla f \cdot \nabla u] d t d a d x \leq C_{3}\left[\int_{Q}\left(u^{2}+|\nabla u|^{2}\right) d t d a d x\right]^{1 / 2} \\
\cdot\left[\int_{Q}\left(w^{2}+|\nabla w|^{2}\right) d t d a d x\right]^{1 / 2}, \\
\int_{Q} \Delta f \cdot \Delta u d t d a d x \leq C_{4}\left[\int_{Q}|\Delta u|^{2} d t d a d x\right]^{1 / 2}\left[\int_{Q}\left(w^{2}+|\nabla w|^{2}+|\Delta w|^{2}\right) d t d a d x\right]^{1 / 2}
\end{gathered}
$$

where:

$$
\begin{aligned}
& C_{3}=2|\mu|_{L^{\infty}(Q)}+|\nabla \mu|_{L^{\infty}(Q)}, \\
& C_{4}=|\mu|_{L^{\infty}(Q)}+|\Delta \mu|_{L^{\infty}(Q)}+2|\nabla \mu|_{L^{\infty}(Q)} .
\end{aligned}
$$

If we substitute (13) and (14) into the estimate (9), using the Schwarz inequality we obtain

$$
\left(\lambda-\frac{C_{3}}{2}\right) \int_{Q}\left[u^{2}+|\nabla u|^{2}\right] d t d a d x \leq \frac{3 C_{1}+C_{3}}{2} \int_{Q}\left[w^{2}+|\nabla w|^{2}\right] d t d a d x .
$$

If we use (13), (14), (10) and the Schwarz inequality we have:

$$
\left(\lambda-\frac{C_{4}}{2}\right) \int_{Q}|\Delta u|^{2} d t d a d x \leq \frac{4 C_{1}+C_{4}}{2} \int_{Q}\left[w^{2}+|\nabla w|^{2}+|\Delta w|^{2}\right] d t d a d x .
$$

Remembering that $\lambda$ was chosen very large compared with $\left(C_{1}, C_{3}, C_{4}\right)$, we easily derive from (16), (17) that there exists a constant $K=K\left(\lambda, C_{1}, C_{3}, C_{4}\right)$ that satisfies $0<K<1$ and such that:

$$
\int_{Q}\left[u^{2}+|\nabla u|^{2}+|\Delta u|^{2}\right] d t d a d x \leq K \int_{Q}\left[w^{2}+|\nabla w|^{2}+|\Delta w|^{2}\right] d t d a d x .
$$

But $w=w^{1}-w^{2}$ and $u=u^{1}-u^{2}=S w^{1}-S w^{2}$. Therefore we proved that $S$ has a unique fixed point. Hence for bounded $\mu$ the problem (1-4) has a unique solution belonging to the space $E$.

4.3 Proof of Theorem 1: Existence. In the general case $\mu$ is not bounded near $a=A$. Let $\lambda$ be a positive constant very large compared with $C_{1}$ and the norms of $\nabla \mu$ and $\Delta \mu$ in $L^{\infty}(Q)$. Once more we deal with $e^{-\lambda t} u$, still denoted $u$. There exists a sequence $\left(\mu_{n}\right)_{n}$ such that:

$$
\begin{aligned}
& \mu_{n} \in L^{\infty}(Q), \\
& \left|\nabla \mu_{n}\right|_{L^{\infty}(Q)}+\left|\Delta \mu_{n}\right|_{L^{\infty}(Q)} \leq C_{5} \text { independent of } n, \\
& \mu_{n}(t, a, x)=\mu(t, a, x) \text { in }(0, T) \times(0, A-1 / n) \times \Omega .
\end{aligned}
$$

For each $n$ there exists a unique $u_{n}$ belonging to $E$, a solution of the problem (I) with $\mu$ changed into $\mu_{n}+\lambda$. But $u_{n}$ is the solution to problem (IV) with:

$$
f=-\mu_{n} u_{n} \text { in } Q, \quad b(t, x)=\int_{0}^{A} \beta(t, a, x) u_{n}(t, a, x) d a \text { in }(0, T) \times \Omega .
$$


Again we use the estimates (9), (10) and we need some preliminary calculations. It is obvious that the inequalities (13) are valid with $u_{n}$ instead of $w$. Now we have:

$$
\int_{Q} f \cdot u_{n}+\nabla f \cdot \nabla u_{n} d t d a d x=-\int_{Q} \mu_{n}\left[u_{n}^{2}+\left|\nabla u_{n}\right|^{2}\right] d t d a d x-\int_{Q} u_{n} \cdot \nabla \mu_{n} \cdot \nabla u_{n} d t d a d x
$$

hence from (18) and Schwarz and Holder inequalities we obtain:

$$
\begin{aligned}
& \int_{Q}\left[f \cdot u_{n}+\nabla f \cdot \nabla u_{n}\right] d t d a d x \\
& \leq-\int_{Q} \mu_{n}\left[u_{n}^{2}+\left|\nabla u_{n}\right|^{2}\right] d t d a d x+\frac{C_{5}}{2} \int_{Q}\left[u_{n}^{2}+\left|\nabla u_{n}\right|^{2}\right] d t d a d x .
\end{aligned}
$$

Along the same lines we transform $\int_{Q} \Delta f \cdot \Delta u_{n} d t d a d x$ into:

$$
-\int_{Q} \mu_{n} \cdot\left|\Delta u_{n}\right|^{2} d t d a d x-\int_{Q}\left[2 \nabla \mu_{n} \cdot \Delta u_{n} \nabla u_{n}+\Delta \mu_{n} \cdot u_{n} \Delta u_{n}\right] d t d a d x
$$

therefore:

$$
\int_{Q} \Delta f \cdot \Delta u_{n} d t d a d x
$$

$$
\leq-\int_{Q} \mu_{n}\left|\Delta u_{n}\right|^{2} d t d a d x+C_{6} \int_{Q}\left[u_{n}^{2}+\left|\nabla u_{n}\right|^{2}+\left|\Delta u_{n}\right|^{2}\right] d t d a d x,
$$

where $C_{6}$ is a constant depending only on $C_{5}$.

Substituting these results in (9) and (10) yields, respectively:

$$
\begin{gathered}
\int_{Q}\left[\lambda+\mu_{n}-\frac{3 C_{1}}{2}-\frac{C_{5}}{2}\right]\left(\left[u_{n}^{2}+\left|\nabla u_{n}\right|^{2}\right] d t d a d x \leq \frac{1}{2} \int_{(0, A) \times \Omega}\left[u_{0}^{2}+\left|\nabla u_{0}\right|^{2}\right] d a d x .\right. \\
\int_{Q}\left[\lambda+\mu_{n}\right]\left|\Delta u_{n}\right|^{2} d t d a d x-C_{6} \int_{Q}\left[\left|u_{n}\right|^{2}+\left|\nabla u_{n}\right|^{2}+\left|\Delta u_{n}\right|^{2}\right] d t d a d x \\
\quad \leq \frac{1}{2} \int_{(0, A) \times \Omega}\left|\Delta u_{0}\right| d a d x .
\end{gathered}
$$

But $\lambda$ has been chosen large. So from (19) and (20) we can deduce that the sequence $\left(u_{n}\right)$ is bounded in the $L^{2}\left(\mathcal{O} ; H^{2}(\Omega)\right)$ norm. The clue to getting this estimate is that $\mu$ is nonnegative. This allows us to remove the hypothesis " $\mu$ bounded."

Hence there exists $u$ in $L^{2}\left(\mathcal{O} ; H^{2}(\Omega)\right.$ and a sub-sequence $\left(u_{n_{p}}\right)_{p}$ (which we simply denote $\left.\left(u_{p}\right)\right)$ such that:

$$
u_{p} \underset{p \rightarrow \infty}{\longrightarrow} u \text { weakly in } L^{2}\left(\mathcal{O} ; H^{2}(\Omega)\right) .
$$

Each $u_{p}$ satisfies the homogeneous Neumann boundary condition; it follows that $u$ satisfies the same homogeneous Neumann boundary condition. 
Now $\left(\Delta u_{n}\right)_{n}$ is bounded in $L^{2}(Q)$; from the equation

$$
\frac{\partial u_{p}}{\partial t}+\frac{\partial u_{p}}{\partial a}+\mu_{p} u_{p}=\Delta \int_{0}^{A} k(a, \alpha) u_{p}(t, \alpha, x) d \alpha \text { in } Q,
$$

we conclude that the sequence $\left(\partial u_{p} / \partial t+\partial u_{p} / \partial a+\mu_{p} u_{p}\right)$ is bounded in the $L^{2}(Q)$-norm. For a subsequence still denoted with the $p$-indices we have:

$$
\frac{\partial u_{p}}{\partial t}+\frac{\partial u_{p}}{\partial a}+\mu_{p} u_{p} \underset{p \rightarrow \infty}{\longrightarrow} h \text { weakly in } L^{2}(Q) .
$$

But the choice of the sequence $\left(\mu_{n}\right)$-see $(18)^{3}$-ensures that:

$$
\frac{\partial u_{p}}{\partial t}+\frac{\partial u_{p}}{\partial a}+\mu_{p} u_{p} \underset{p \rightarrow \infty}{\longrightarrow} \frac{\partial u}{\partial t}+\frac{\partial u}{\partial a}+\mu u \text { in } \mathscr{D}^{\prime}(Q),
$$

that is, in the distributional sense in $Q$. Thus $h=u_{t}+u_{a}+\mu u$ and, letting $p \rightarrow+\infty$ in (21), we obtain that $u$ satisfies Eq. (1).

We may notice that weakly in $\left.L^{2}((0, T) \times \Omega)\right)$ we have:

$$
u_{p}(t, 0, x)=\int_{0}^{A} \beta(t, a, x) u_{p}(t, a, x) d a \underset{p \rightarrow \infty}{\longrightarrow} \int_{0}^{A} \beta(t, a, x) u(t, a, x) d a .
$$

Let $A_{0}$ be such that $0<A_{0}<A$ and define $\mathcal{O}_{0}=(0, T) \times\left(0, A_{0}\right)$; for $n \geq n\left(A_{0}\right) \mu_{n}(t, a$, $x)=\mu(t, a, x)$ in $Q_{0}$. Hence we have $\mu \in L^{\infty}\left(Q_{0}\right)$ and from Eq. (21) we get $\left(\partial u_{p} / \partial t\right.$ $\left.+\partial u_{p} / \partial a\right)_{p}$ bounded in $L^{2}\left(Q_{0}\right)$. Thus:

$$
\begin{aligned}
& u_{p} \underset{p \rightarrow \infty}{\longrightarrow} u \text { weakly in } L^{2}\left(Q_{0}\right), \\
& \frac{\partial u_{p}}{\partial t}+\frac{\partial u_{p}}{\partial a} \longrightarrow \frac{\partial u}{\partial t}+\frac{\partial u}{\partial a} \text { weakly in } L^{2}\left(Q_{0}\right),
\end{aligned}
$$

and by continuity it follows that:

$$
\begin{aligned}
& u_{p}(0, a, x) \underset{p \rightarrow \infty}{\longrightarrow} u(0, a, x) \text { weakly in } L^{2}\left(\left(0, A_{0}\right) \times \Omega\right), \\
& u_{p}(t, 0, x) \underset{p \rightarrow \infty}{\longrightarrow} u(t, 0, x) \text { weakly in } L^{2}(0, T) \times \Omega .
\end{aligned}
$$

From (22) we deduce that $u$ satisfies the initial condition (2)). For each $u_{p}$ verifying (4) on $(0, A) \times \Omega$ we have:

$$
u(0, a, x)=u_{0}(a, x) \text { in }\left(0, A_{0}\right) \times \Omega ;
$$

this equality being true for any $A_{0}$ lying in $(0, A)$ is true in the open set $(0, A) \times \Omega$. So the initial condition (4) is fulfilled.

We can also derive that $\mu^{1 / 2} u$ belongs to $L^{2}\left(\mathcal{O} ; H^{2}(\Omega)\right)$.

4.4 Proof of Theorem 1: Uniqueness. Let $\lambda$ be large enough with respect to $C_{1}$; we perform the change of unknown function $u(t, a, x)=e^{\lambda t} v(t, a, x)$ and we deal with $v$ which we simply denote $u$.

Let $u$ be in $L^{2}\left(\mathcal{O} ; H^{2}(\Omega)\right.$ ), a solution to problem (I), and satisfy the homogeneous Neumann condition on $\mathcal{O} \times \partial \Omega$, and let $u(0, a, x)=0$ in $(0, A) \times \Omega$. 
For any $A_{0}$ such that $0<A_{0}<A$ we let $Q_{0}=(0, T) \times\left(0, A_{0}\right) \times \Omega$. Multiplying Eq. (1) by $u$ and integrating over $Q_{0}$ yields:

$$
\begin{aligned}
\int_{Q_{0}}\left(u_{t}+u_{a}+\mu u+\lambda u\right) u d t d a d x & \\
& -\int_{Q_{0}}\left[\Delta \int_{0}^{A} k(a, \alpha) u(t, \alpha, x) d \alpha\right] u d t d a d x=0 .
\end{aligned}
$$

We already know that $u_{t}+u_{a}$ lie in $L^{2}\left(Q_{0}\right)$ because $\mu \in L^{\infty}\left(Q_{0}\right)$. So we can integrate by parts the first term in the left-hand side of (23); we obtain, using (2) and $u(0, a, x)=0$ :

$\frac{1}{2} \int_{\left(0, A_{0}\right) \times \Omega} u^{2}(T, a, x) d a d x+\frac{1}{2} \int_{\left(0, A_{0}\right) \times \Omega} u^{2}\left(t, A_{0}, x\right) d t d x$

$$
+\int_{Q_{0}}(\lambda+\mu) u^{2} d t d a d x-\frac{1}{2} \int_{(0, T) \times \Omega}\left[\int_{0}^{A} \beta \cdot u d a\right]^{2} d t d x .
$$

Moreover, we can integrate by parts the second integral in the left-hand side of (23). These two calculations lead to the estimate:

$\lambda \int_{Q_{0}} u^{2}(t, a, x) d t d a d x$

$$
\begin{aligned}
+\int_{(0, T) \times \Omega}\left[\nabla \int_{0}^{A} k(a, \alpha) u(t, \alpha, x) d \alpha \cdot \nabla \int_{0}^{A_{0}} u(t, a, x) d a\right] d t d x & \\
& \leq \frac{1}{2} \int_{(0, T) \times \Omega}\left[\int_{0}^{A} \beta u d a\right]^{2} d t d x .
\end{aligned}
$$

So if we let $A_{0} \rightarrow A$, using the properties of the kernel $k$ we get

$$
\lambda \int_{Q} u^{2} d t d a d x \leq \frac{1}{2} \int_{(0, T) \times \Omega}\left[\int_{0}^{A} \beta u d a\right]^{2} d t d x .
$$

From the condition $(\beta)^{1}$ we derive:

$$
\left(\lambda-\frac{C_{1}}{2}\right) \int_{Q} u^{2} d t d a d x \leq 0 \text { and } u=0 \text { in } Q .
$$

4.5 Sketch of the proof of Theorem 3. We must modify the first step, because (9), (10) are not necessarily fulfilled when we change $(k)^{1}$ into $(k)^{2}$.

When $(\mathrm{k})^{2}$ is satisfied the analogue to problem (IV) is:

$$
\begin{aligned}
& u_{t}+u_{a}+\lambda u-k(a) \Delta \int_{0}^{A} u(t, a, x) d a=f \text { in } Q, \\
& u(0, a, x)=u_{0}(a, x) \text { in }(0, A) \times \Omega, \\
& u(t, 0, x)=b(t, x) \text { in }(0, T) \times \Omega, \\
& \frac{\partial}{\partial \eta} \int_{0}^{A} u(t, a, x) d a=0 \text { in } \mathcal{O} \times \partial \Omega .
\end{aligned}
$$


Let $Q_{T}=(0, T) \times \Omega$. Assuming:

$$
\begin{gathered}
f \in L^{2}(Q), \quad u_{0} \in L^{2}((0, A) \times \Omega), \\
\int_{0}^{A} u_{0}(a, x) d a \in H^{1}(\Omega), \quad b \in L^{2}((0, A) \times(\Omega)),
\end{gathered}
$$

then any $u$ in $L^{2}(Q)$ verifying (6), that is:

$$
\int_{0}^{A} u(t, a, x) d a=P(t, x) \in L^{2}\left(0, T ; H^{2}(\Omega)\right),
$$

and a solution to problem $(\mathrm{V})$ is such that:

$$
\begin{gathered}
P_{t}-K \Delta P+\lambda P=\int_{0}^{A} f(t, a, x) d a+b(t, x)-u(t, A, x) \text { in } Q_{T}, \\
P(0, x)=\int_{0}^{A} u_{0}(a, x) d a=p_{0}(x) \text { in } \Omega, \\
\partial P / \partial \eta=0 \text { in }(0, T) \times \partial \Omega,
\end{gathered}
$$

where we have used $k=\int_{0}^{A} k(a) d a$. This is obtained by integrating Eq. $(\mathrm{V})^{1}$ with respect to the variable $a$. The hypotheses on $\left(f, b, u_{0}\right)$ and $(u, P)$ are consistent. Now $u$ is the solution to:

$$
\begin{gathered}
u(0, a, x)=u_{0}(a, x) \text { in }(0, A) \times \Omega, \\
u(t, 0, x)=b(t, x) \text { in }(0, T) \times \Omega, \\
u_{t}+u_{a}+\lambda u=k(a) \Delta P+f \text { in } Q .
\end{gathered}
$$

If $u$ belongs to $L^{2}(Q)$ and satisfies (6) and if $\left(f, b, u_{0}\right)$ satisfies (24), a priori bounds can be derived from Eq. (VI) and (VII).

For the parabolic equation (VI) we have:

$$
\begin{aligned}
\frac{1}{2} \int_{\Omega} \mid \nabla P(T, & x)\left.\right|^{2} d x+\int_{Q_{T}}\left[\lambda|\nabla P|^{2}+K|\Delta P|^{2}\right] d t d x \\
& =\frac{1}{2} \int_{\Omega}\left|\nabla p_{0}\right|^{2} d x+\int_{Q_{T}}\left[\int_{0}^{A} f(t, a, x) d a+b-u(t, A, x)\right][-\Delta P] d t d x .
\end{aligned}
$$

As for the first-order equation (VII), integrating by parts yields:

$$
\begin{aligned}
\frac{1}{2} \int_{Q} u^{2}(t, A, x) d t d x+\lambda \int_{Q} u^{2} d t d a d x \leq & \int_{Q}[f-k(a) \Delta P] u d t d a d x \\
& +\frac{1}{2} \int_{Q T} b^{2}(t, x) d t d x+\frac{1}{2} \int_{(0, A) \times \Omega} u_{0}^{2} d a d x .
\end{aligned}
$$

Employing the method of separation of variables and (25), (26) we prove for problem (V) the analogue to Theorem 4. Via a fixed-point method we get Theorem 3 for bounded $\mu$. It suffices to use a suitable linear combination of (25) and (26). 
Now we want to turn to the general case, namely, the case when $\mu$ is not bounded. $\mu$ can be approximated by a sequence $\left(\mu_{n}\right)_{n}$ satisfying (18); for each integer there is a unique $u_{n}$ in $L^{2}(Q)$ verifying (6) and solution to problem (II); that is, $u_{n}$ and $P_{n}(t, x)=\int_{0}^{A} u(t, a, x)$ $d a$ are solutions to (VI) and (VII) with:

$$
f=-\mu_{n} u_{n} \text { in } Q, \quad b=\int_{0}^{A} \beta u_{n} d a \text { in }(0, T) \times \Omega .
$$

From (26) we can get an estimate on $\left(\mu_{n}^{1 / 2} \cdot u_{n}\right)_{n}$ in $L^{2}(Q)$ but not on the term $-\int_{0}^{A} \mu_{n} \cdot u_{n} d a$ that appears on the right-hand side of (25) when $f$ is given by (27). However, when the condition $(\mu)^{5}$ is satisfied we have:

$$
\left|\int_{0}^{A} \mu_{n} \cdot u_{n} d a\right| \leq \int_{0}^{A} \mu_{n}^{1 / 2} \mu_{n}^{1 / 2}\left|u_{n}\right| d a \leq m^{1 / 2} \cdot\left(\int_{0}^{A} \mu_{n} u_{n}^{2} d a\right)^{1 / 2} .
$$

This is enough to obtain that $\left(u_{n}\right)$ and $\left(\mu_{n}^{1 / 2} \cdot u_{n}\right)$ (resp. $\left.P_{n}\right)$ are bounded in $L^{2}(Q)$ (resp. $\left.L^{2}\left(0, T ; H^{2}(\Omega)\right)\right)$ and to prove Theorem 3 by letting $n \rightarrow \infty$.

When $(\mu)^{5}$ is not fulfilled we shall derive from $(\mu)^{4}$ that $\left(\mu_{n} u_{n}\right)$ is bounded in the $L^{2}(Q)$-norm. If we multiply Eq. (VII) that $u_{n}$ satisfies by $\mu_{n}^{2} u_{n}$ and if we integrate over $Q$ we obtain:

$$
\begin{aligned}
\int_{Q}\left[\mu_{n}^{2}+\right. & \left.\lambda \mu_{n}-\frac{\partial \mu_{n}}{\partial t}-\frac{\partial \mu_{n}}{\partial a}\right] \mu_{n} u_{n}^{2} d t d a d x \\
\leq & \frac{1}{2} \int_{(0, A) \times \Omega}\left[\mu_{n}^{2}(0, a, x) u_{0}^{2}(a, x) d a d x+\frac{1}{2} \int_{Q_{T}} \mu_{n}^{2}(t, 0, x)\left[\int_{0}^{A} \beta u_{n} d a\right]^{2} d t d x\right. \\
& -\int_{Q}\left[\mu_{n} k \Delta P_{n}\right] \cdot\left[\mu_{n} u_{n}\right] d t d a d x .
\end{aligned}
$$

We can choose the sequence $\left(\mu_{n}\right)$ so that each $\mu_{n}$ satisfies $(\mu)^{4}$ uniformly with respect to $n$. Thus:

$$
\begin{aligned}
& \left(\lambda+\lambda_{1}\right) \int_{Q} \mu_{n}^{2} u_{n}^{2} d t d a d x+\lambda_{2} \int_{Q} \mu_{n} u_{n}^{2} d t d a d x \\
& \quad \leq C_{7}+C_{8} \int_{Q} u_{n}^{2} d t d a d x+M^{1 / 2} \cdot\left[\int_{Q_{T}}\left|\Delta P_{n}\right|^{2} d t d x\right]^{1 / 2}\left[\int_{Q} \mu_{n}^{2} u_{n}^{2} d t d a d x\right]^{1 / 2}
\end{aligned}
$$

where $C_{7}$ and $C_{8}$ are independent of $n$. From (28), (25) and (26) are satisfied by $u_{n}$ and $P_{n}$ when $(f, b)$ is given by $(27)$ and the inequality

$$
\left|\int_{0}^{A} \mu_{n} u_{n} d a\right| \leq A^{1 / 2}\left(\int_{0}^{A} \mu_{n}^{2} u_{n}^{2} d a\right)^{1 / 2} .
$$

We derive that if $\lambda$ has been chosen large enough:

$$
\int_{Q}\left[u_{n}^{2}+\mu_{n}^{2} u_{n}^{2}\right] d t d a d x+\int_{Q_{T}}\left|\Delta P_{n}\right|^{2} d t d x \leq C_{9} \text { independent of } n .
$$

So letting $n \rightarrow \infty$ we conclude. 
Remark 3. An alternative method is to approximate the kernel $k$ in such a way that the condition (k) ${ }^{4}$ is satisfied (see Sec. 3 ).

\section{BiBLIOGRAPHY}

[1]. G. diBlasio and L. Lamberti, An initial boundary value problem for age population diffusion, SIAM J. Appl. Math. 35, 530-615(1978)

[2] M. G. Garroni and M. Langlais, Age-dependent population diffusion with external constraints, J. Math. Biol., to appear

[3] M. E. Gurtin, A system of equations for age-dependent population diffusion, J. Theor. Biol. 40 389-392 (1973)

[4] M. E. Gurtin and R. C. MacCamy, On the diffusion of biological population, Math. Biosci. 38, 35-49 (1977)

[5] M. E. Gurtin and R. C. MacCamy, Some simple models for nonlinear age-dependent population dynamics, Math. Biosci. 43, 199-211 (1979)

[6] F. Hoppensteadt, Mathematical theories of populations: demographics, genetics, and epidemics, Soc. Ind. Appl. Math., Philadelphia (1975)

[7] H. L. Langhaar, General population theory in age-time continuum, J. Franklin Inst. 293, 199-214 (1972)

[8] M. Langlais, Sur un problème de dynamique de population, Tech. Rep. A. A. I., University of Bordeaux 1 (1980)

[9] M. Langlais, Thesis, University of Bordeaux 1 (1981)

[10] J. L. Lions and E. Magènes, Problèmes aux limites homogènes et applications, tôme 1, Dunod, Paris (1968)

[11] R. C. MacCamy, A population model with nonlinear diffusion, J. Diff. Eqs. 39, 52-72 (1981) 